\begin{tabular}{|l|l|c|c|}
\hline Eiszeitalter u. Gegenwart & 30 & $\begin{array}{c}161-170 \\
2 \mathrm{Abb} .\end{array}$ & Hannover 1980 \\
\hline
\end{tabular}

\title{
Die Höhenlage ur- und frühgeschichtlicher Wohnniveaus in nordwestdeutschen Marschengebieten als Höhenmarken ehemaliger Wasserstände
}

\begin{abstract}
Klaus BRANDT *)
Marshlands, prehistory, early history, habitation, height level, indicator, water level, river, Holocene. North West German Lowlands (Ems and Elbe River)

$\mathrm{K} u \mathrm{rzf}$ assung: In Form eines Zeit-Höhen-Diagramms werden die Höhenlagen ur- und frühgeschichtlicher Wohnniveaus in der Marsch zwischen Ems- und Elbemündung dargestellt. Es werden die Faktoren diskutiert, die bewirken, daß die gemessene Höhenlage der Wohnniveaus von der ursprünglichen Höhenlage abweicht. Die Wohnnıveaus können als Marken für Höchstwasserstände gelten, und zwar sind die Wohnniveaus nur bestimmter Siedlungsschichten aussagekräftig, vor allem solcher, die einen Neubeginn der Besiedlung in einer Flachsiedlung oder auf einem Wurtauftrag markieren. Das Diagramm spiegelt die drei Siedlungsperioden in der nordwestdeutschen Marsch wider. Die Flachsiedlungen jeweils zu Beginn dieser Siedlungsperioden markieren einen Stillstand im Anstieg des Wasserspiegels. Die Wohnniveaus in gleichaltrigen Marschensiedlungen weichen in ihren Höhenlagen z.T. stark voneinander ab, da das Hochwasser in unbedeichter Marsch unterschiedlich hoch auflief, je nachdem, wie weit die Entternung zum offenen Meer war.
\end{abstract}

\section{[The Elevation of Prehistoric and Early Historic Habitation in the Marshlands of Northwestern Germany as Height Indicators or Former Water Levels]}

A bstract: The elevation of prehistoric and early historic habitation in the marshlands between the estuaries of the Ems and Elbe rivers are presented in a time-altitude graph. Keasons for the difference between the present and original elevations are discussed. Habitation levers may be regarded as index marks of the highest water level, but only those habitation levels, whicn mark the beginning of a settlement on tlat marshy ground or on a new layer of a dwellnng mouna. Three periods of settlement in the marshlands of northwestern Germany are shown by tue grapn. Settlements on flat ground mark a standstill during Holocene sea-level rise. Habitation teveis oi the same age often have different elevations, because in the undiked marshlands the tidal range varies according to the distance to the sea.

\section{Einführung}

In den vergangenen Jahren haben archäologische Grabungen in der nordwestdeutschen Marsch eine große Menge von neuen Beobachtungen geliefert, so daß es sinnvoll erscheint, die Höhenlagen archäologisch datierter Wohnniveaus in den Marschen zwischen Ems- und Elbemündung zusammenzustellen. Vor allem sind es Untersuchungen des Niedersächsischen Landesinstituts für Marsch- und Wurtenforschung, Wilhelmshaven, gewesen, die neues Material geliefert haben. Allen Kollegen und Mitarbeitern am Niedersächsischen Landesinstitut für Marschen- und Wurtenforschung danke ich für ihre vielfältige Hilfe. Von großem Nutzen war insbesondere das unveröffentlichte Manuskript eines V ortrages von W. HaARnAGel „Die Besiedlung im nordwestdeutschen Küstengebiet in ihrer Abnangigkeit von Meeresspiegelschwankungen und Sturmfluten“ (1978). Die folgende Zusammenstellung ist ein erster Schritt auf dem Wege, die in Grabungen eingemessenen Höhenlagen von Siedlungsspuren auszuwerten im Hinblick auf die Frage der Bewegungen des Nieereswasserspiegels während des Holozän.

*) Anschrift des Verfassers: Dr. K. B r a n d t , Niedersächsisches Landesinstitut für Marschenund Wurtenforschung, Viktoriastraße 26/28, D-2940 Wilhelmshaven. 


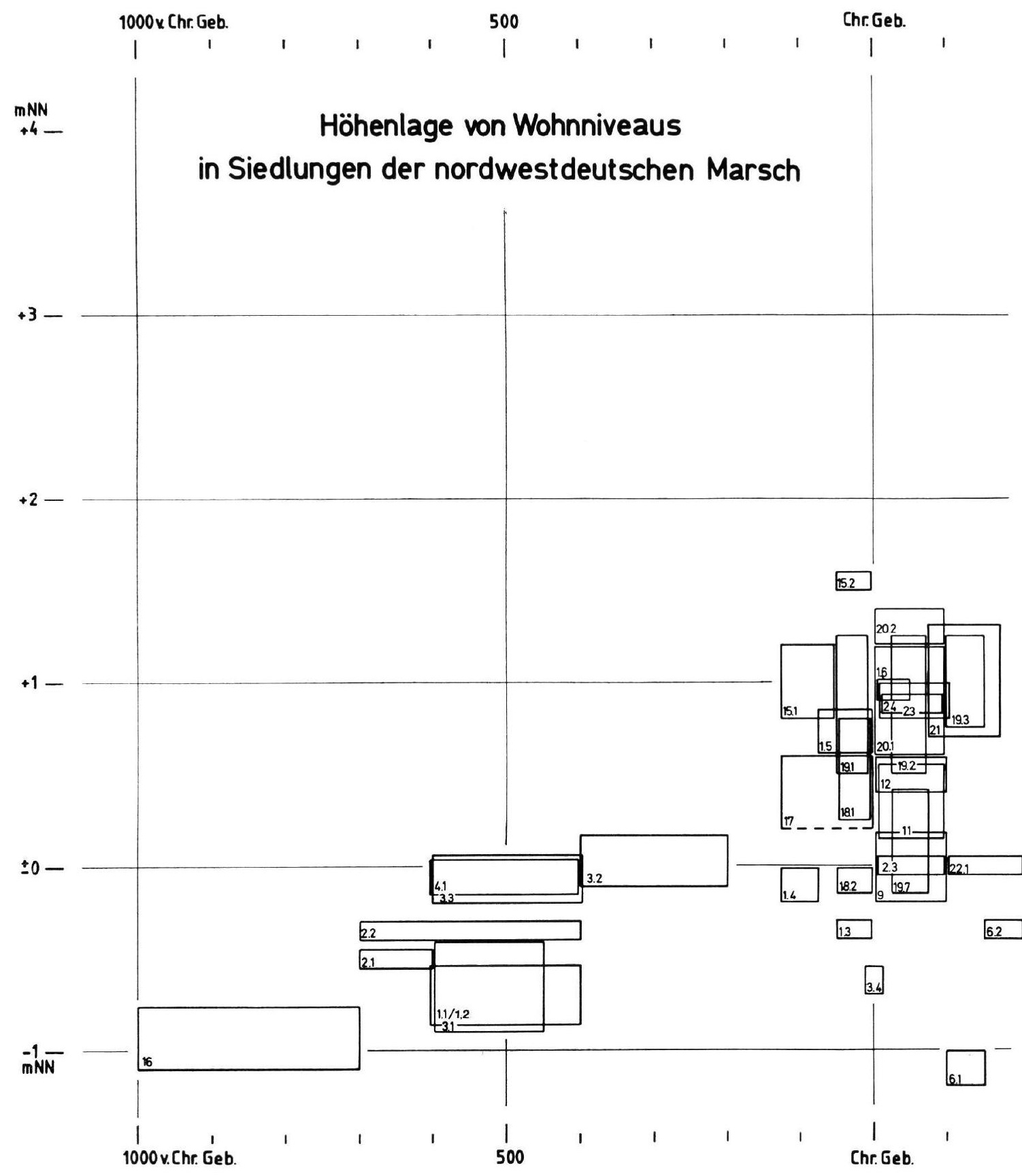

Abb. 1: Höhenlagen von Wohnniveaus in ur- und frühgeschichtlichen Siedlungen in der Marsch zwischen Ems- und Elbemündung. 


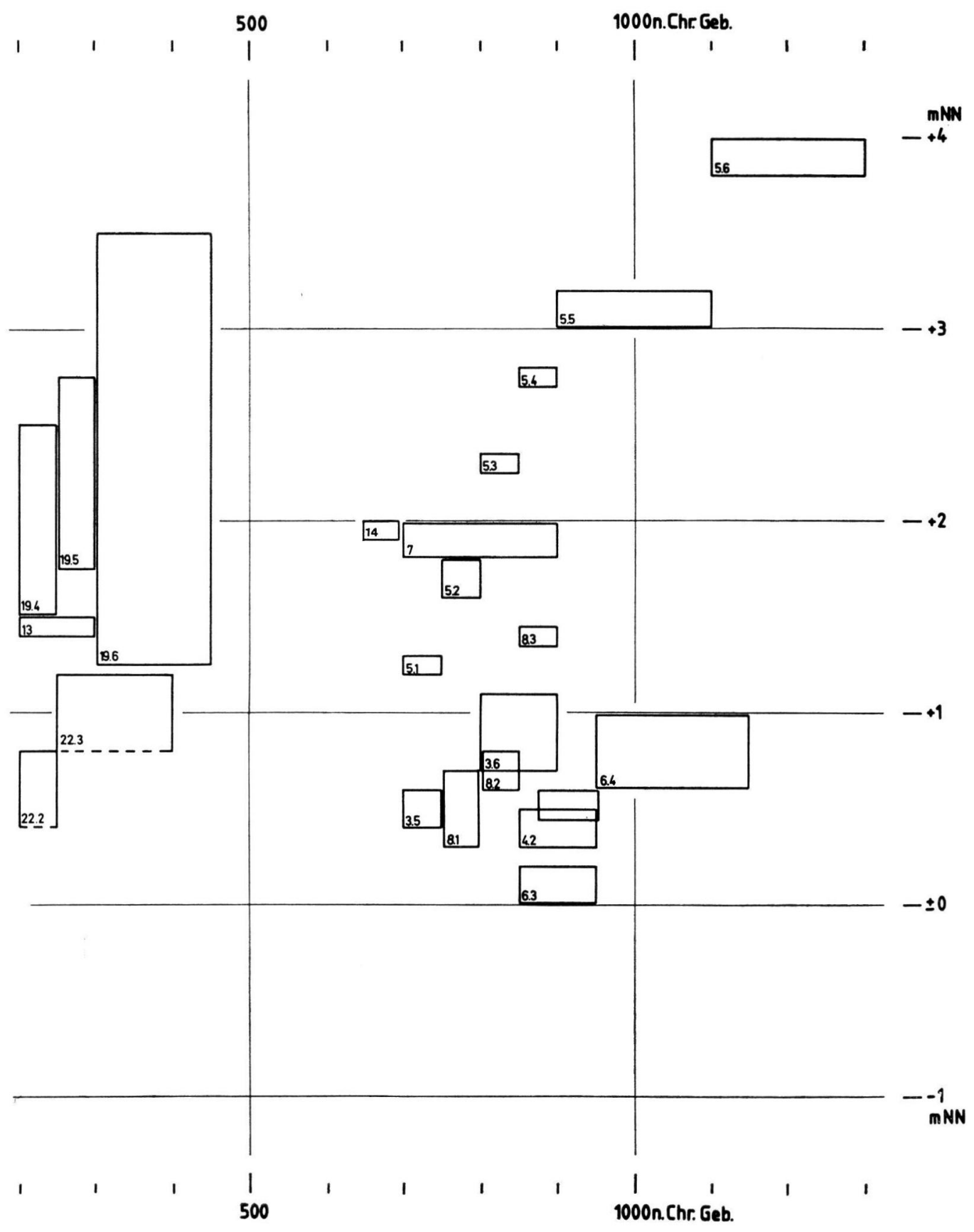




\section{Methodische Bemerkungen zur Darstellung und Auswertung der Höhenlagen ur- und frühgeschichtlicher Wohnniveaus}

Die gemessene Höhenlage kann von der ursprünglichen Höhenlage mehr oder weniger stark abweichen. Die Ursachen für solche Unterschiede sind in Setzung, Tektonik und Isostasie zu suchen (Sindowski \& Streif 1974; Louwe-Kooljmans 1976). Die Auswirkungen von tektonischer und isostatischer Senkung sind schwer zu bestimmen. Es wird im Nordseeküstengebiet mit Werten zwischen 1,7 und $5 \mathrm{~cm} / J$. gerechnet. Jedoch stellen die genannten Senkungsbeträge Durchschnittswerte für den Zeitraum seit dem Holstein- bzw. Eem-Interglazial dar. Genauere Angaben darüber, wann die Senkung innerhalb des genannten Zeitraumes erfolgte, lassen sich nicht machen, so daß für den hier diskutierten Zeitraum der letzten 3000 Jahre zwar grundsätzlich mit tektonischer und isostatischer Senkung zu rechnen ist, genaue Werte aber nicht zur Verfügung stehen.

Außer Tektonik können auch Setzungsvorgänge bewirken, daß die gemessene Höhenlage nicht der ursprünglichen entspricht. Im Prinzip unterliegen alle jungen Sedimente der Setzung, vor allem Torfe und in geringerem Maße frischer Klei; bei Sand ist der Setzungsbetrag so unerheblich, daß er vernachlässigt werden kann. Aus diesem Grunde sind alle jene ur- und frühgeschichtlichen Siedlungen nicht berücksichtigt worden, wo im Untergrund mächtigere Torfschichten vorhanden sind. Die im folgenden ausgewerteten Wohnniveaus liegen auf Strand- oder Uferwällen, die aus schluffigen bis sandigen Tonen aufgebaut sind. Da zwischen der Ablagerung dieser Sedimente und ihrer Besiedlung eine gewisse Zeit verstrichen ist, können in der Zeit nach der Besiedlung stärkere Setzungen ausgeschlossen werden.

Es kann hier nicht diskutiert werden, inwieweit im Einzelfall Tektonik und Setzung sich auf die heutige Höhenlage der ur- und frühgeschichtlichen Wohnniveaus ausgewirkt haben. Jedoch zeigen die unterschiedlich hohen Wohnniveaus mittelalterlicher Siedlungen in der Krummhörn (vgl. Abb. 1: Groothusen (5) und Visquard (7) mit Alt-Damhusen (6.3-6.4) und Middelstewehr (8) ), daß dort Sackung und vielleicht auch Tektonik eine Rolle spielen (Wildvang 1938; Streif 1971; Sindowski \& Streif 1974).

Die Wohnniveaus innerhalb ur- und frühgeschichtlicher Siedlungen stellen eine klar definierte Höhenlage in Bezug auf die jeweils herrschenden Wasserstände dar. Bei den Höhenangaben der Wohnniveaus wurden nur die Teile einer Siedlung berücksichtigt, in denen Häuser gestanden haben. Die Gehniveaus in den Häusern dürften so hoch gelegen haben, daß sie vor Überflutung sicher waren. Da diese Häuser ganz überwiegend in bäuerlichen Siedlungen lagen, wurden im Hinblick auf die Sicherheit vor Überflutungen überall dieseiben Anforderungen an die Höhenlage gestellt. Ausnahmen könnten Siedlungen gebilcet haben, wo Bewohner sich aus wirtschaftlichen Gründen in ungünstigeren Lagen ansiedelten, z. B. an Schiffslandeplätzen, wo sich ein guter Zugang zum schiffbaren Gewässer bot und deshalb häufigere Überflutungen als sonst in Kauf genommen wurden.

In der Siedlung Bentumersiel (1.3), einem Warenumschlagplatz der Zeit um Chr. Geb., könnten diese Gesichtspunkte eine Rolle gespielt haben (BRANDT 1977), so daß sich das tiefgelegene Wohnniveau der Häuser von Bentumersiel dadurch erklären ließe. Bei den Schiffsanlegern der Siedlungen Boomborg (3.4) und Feddersen Wierde (19. 7) ergibt sich die niedrige Lage aus der Funktion der Anlagen.

Bei der Durchsicht der verwertbaren Befunde von größeren Siedlungsgrabungen in der Marsch zeigte sich sehr bald, daß in Wurtsiedlungen gleichaltrige Häuser verschieden hoch lagen. Am aussagekräftigsten ist die Höhenlage des am niedrigsten gelegenen Hauses. Es werden in diesen Fällen aber die Wohnniveaus aller Häuser berücksichtigt. Denn sonst ergäben sich Schwierigkeiten, wenn Befunde aus größeren Grabungen mit denen aus kleineren Grabungen verglichen würden. Bei Suchgräben oder Grabungsflächen geringer Ausdehnung in einer größeren Siedlung ist in der Regel schwer zu entscheiden, ob die auf- 


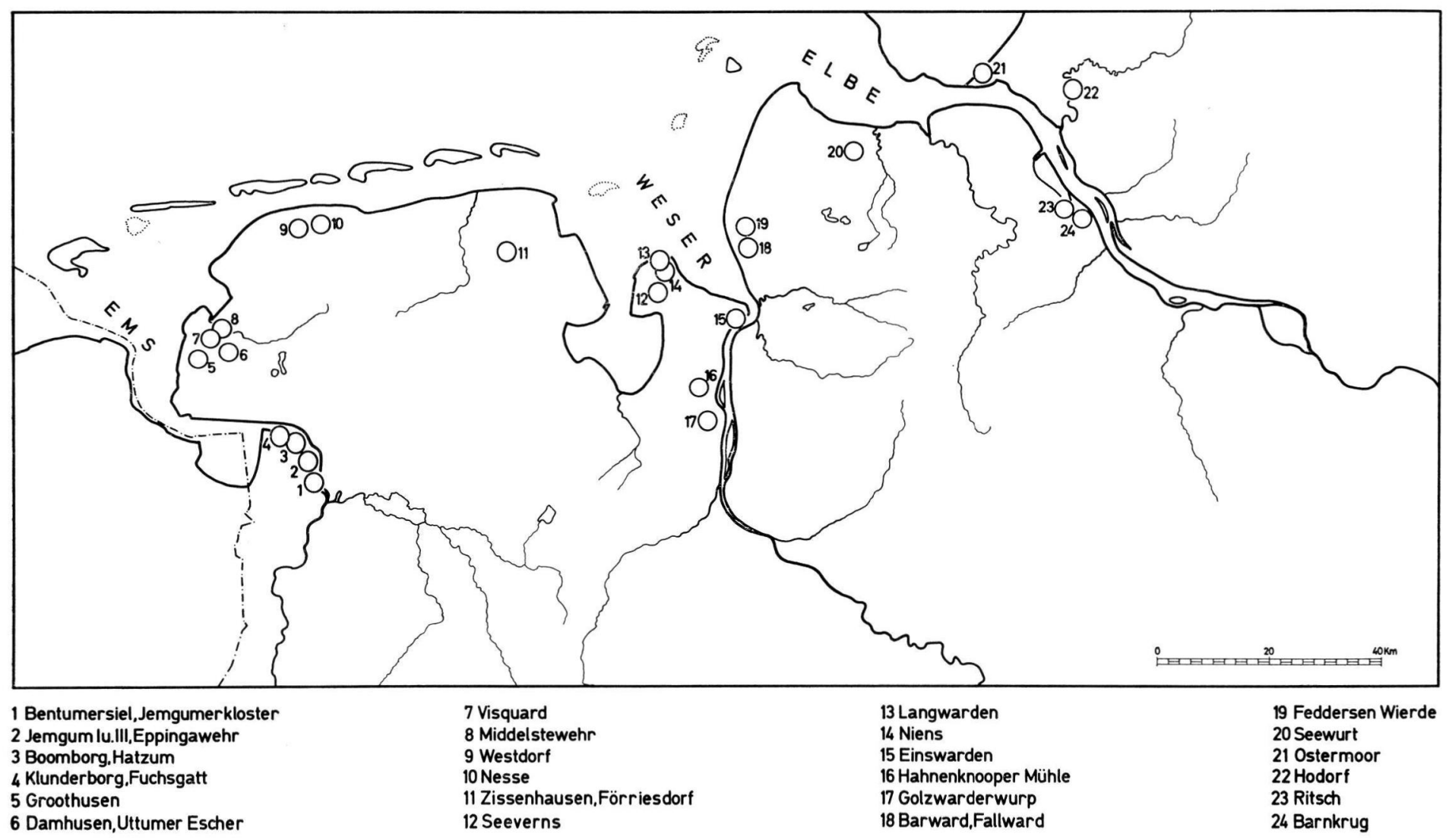

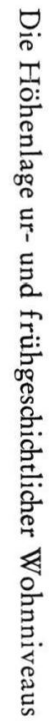

Abb. 2: Ur- und frühgeschichtliche Siedlungen in der Marsch zwischen Ems- und Elbemündung, in denen die Höhenlage von Wohnniveaus bekannt ist. 
gedeckten Häuser im Verhältnis zu anderen gleichaltrigen Häusern hoch oder niedrig lagen.

Kommen in einer ur- und frühgeschichtlichen Siedlung mehrere Siedlungshorizonte übereinander vor, dann sind für die hier anstehenden Fragen nur diejenigen Horizonte von Belang, die einen Neubeginn der Besiedlung markierten, also darüber etwas aussagen, wie hoch ein Gelände liegen mußte, damit es zu besiedeln war. In einer Flachsiedlung ist das die älteste Siedlungsschicht. Die jüngeren Häuser wurden oft dort errichtet, wo vorher bereits auch Gebäude gestanden hatten und das Wohnniveau durch Siedlungsabfall und Abbruchmaterial der Vorgängerbauten etwas angehoben war. Infolgedessen bleibt in diesen Fällen unklar, ob sich in dem höheren Wohnniveau ein Anstieg des Wasserstandes widerspiegelt. Deshalb bleiben die Wohnniveaus der jüngeren Stadien von Flachsiedlungen hier außer Betracht. In Wurtsiedlungen werden nur die Wohnniveaus berücksichtigt, die einer Wurtauftragsschicht unmittelbar aufliegen. In einem Diagramm (Abb. 1) wurden die Höhenlagen der ur- und frühgeschichtlichen Wohnniveaus eingetragen, so daß die Höhenlage der Wohnniveaus zu m NN (auf der senkrechten Achse) und die Dauer der Besiedlung (auf der waagerechten Achse) ablesbar sind. In einigen Fällen (z. B. 16 oder 2.2) könnte die Darstellung zu der Annahme führen, daß eine Siedlung mehrere Jahrhunderte lang bestand und immer in ein und demselben Niveau gesiedelt wurde. In Wirklichkeit aber bestanden die Siedlungen nicht so lange, es ist aufgrund der vorliegenden Datierungen aber nicht möglich, die Siedlungsdauer genauer einzugrenzen. Bei der Datierung der Siedlungen der älteren Eisenzeit, wurden die neueren Arbeiten zur Chronologie dieser Periode berücksichtigt (vor allem Waterbolk \& Boersma 1976 und LöberT).

Die Wohnniveaus sind mit Zahlen bezeichnet, und zwar in der Reihenfolge von Westen nach Osten wie die Siedlungsplätze im Küstengebiet sich aneinander reihen, ohne Rücksicht auf das Alter der Siedlung. Um die Karte der Fundplätze (Abb. 2) übersichtlich zu halten, wurden eng benachbarte Siedlungen unter einer Zahl zusammengefaßt und die einzelnen Siedlungsplätze und ihre verschiedenen Wohnniveaus durch eine zweite Zahl gekennzeichnet (vgl. Verzeichnis der Wohnniveaus in ur- und frühgeschichtlichen Siedlungen in der Marsch zwischen Ems- und Elbemündung).

\section{Ergebnisse der Auswertung}

Das Diagramm zeigt zunächst einmal, daß im Marschengebiet zwischen Ems- und Elbemündung während der vergangenen 3000 Jahre Zeiträume dichter Besiedlung abwechselten mit Zeitabschnitten sehr schwacher Besiedlung, wenn nicht sogar völliger Entsiedlung. Allerdings ist zu berücksichtigen, daß bei weitem nicht alle ur- und frühgeschichtlichen Siedlungen in das Diagramm aufgenommen wurden, sondern nur diejenigen, aus denen genau eingemessene Wohnniveaus bekannt sind. Deshalb kann hier die Frage der Siedlungskontinuität nicht erörtert werden. Doch zeichnen sich auch in dem Diagramm die drei Zeitabschnitte mit dichter Besiedlung deutlich ab, erstens die jüngere Bronzezeit und ältere vorrömische Eisenzeit bis zum 3. Jh. v. Chr. Geb., zweitens der Zeitraum von der Spätlatènezeit bis zur frühen Völkerwanderungszeit (etwa 100 v. Chr. Geb. bis Mitte 5. Jh. n. Chr. Geb.) und drittens die mit dem 7./8. Jh. n. Chr. Geb. einsetzende Siedlungsperiode. Während der ältesten Siedlungsperiode und jeweils zu Beginn der beiden jüngeren Perioden konnte in der Marsch zu ebener Erde gesiedelt werden, waren also die höher aufgelandeten Teile der Marsch vor Überflutungen im großen und ganzen sicher.

Diese Flachsiedlungen markieren Zeiten niedriger Höchstwasserstände; voraufgegangen waren jeweils Zeitabschnitte mit höher auflaufendem Wasser, vor allem höheren Sturmfluten, durch die in den später besiedelten Gebieten Sedimente abgelagert wurden. Die Flachsiedlungen zeigen zwar einen Stillstand im Anstieg des Wasserspiegels an, geben aber nicht den Beginn der Stillstandsphasen an. Vielmehr ist davon auszugehen, daß die 
Besiedlung der Marsch erst erfolgte, nachdem sich die frisch sedimentierten Ablagerungen $\mathrm{zu}$ wirtschaftlich nutzbarer Marsch entwickelt hatten.

Der Wiederbeginn der Besiedlung in der Zeit um Chr. Geb. vollzog sich in der nordwestdeutschen Marsch durchaus nicht überall gleichzeitig. Die zeitliche Staffelung über einen Zeitraum von etwa zwei Jahrhunderten ist durch die Ergebnisse umfangreicher Grabungen gesichert. Die älteste Gruppe unter diesen Siedlungen bilden die Flachsiedlungshorizonte von Jemgumkloster (1.4), Einswarden (15.1) und Golzwarderwurp (17), die um 100 v. Chr. Geb. besiedelt wurden. Die jüngsten sind die Siedlungen von Ostermoor (21) und Hodorf (22.1). Die ältesten Siedlungen dieser Gruppe liegen im Gebiet westlich der Weser, die jüngsten im Elbegebiet. Daß dies nicht Zufall ist, zeigt ein Blick auf die Marschen der niederländischen Provinzen Groningen und Friesland. Dort gibt es Siedlungen, die vom 6./5. Jh. v. Chr. Geb. durchgehend bis in die ersten Jahrhunderte n. Chr. Geb. bewohnt waren (WATERBOLK \& Boersma 1976). Es ist zu vermuten, daß die zeitliche Staffelung der Wiederbesiedlung um Chr. Geb. mit einer regionalen und zeitlichen Differenzierung in der natürlichen Entwicklung des nordwestdeutschen Küstengebiets zusammenhängt.

Im Verlauf der zweiten und dritten Siedlungsperiode wurden einige Siedlungen aufgegeben, viele Siedlungsplätze aber wurden zu Wurten aufgehöht. In den immer stärker angehobenen Wohnniveaus der Wurtsiedlungen dokumentiert sich der erneute Anstieg der Wasserstände nach der Stillstandsphase.

So klar das Diagramm die drei Perioden in der Siedlungsentwicklung des Marschengebietes zwischen Ems- und Elbemündung widerspiegelt, ergibt sich im einzelnen doch ein sehr differenziertes Bild. Zur selben Zeit bewohnte Siedlungen lagen in unterschiedlich hohen Niveaus. Es kommen Höhenunterschiede von über $1 \mathrm{~m}$ vor. Es liegt auf der Hand, daß dabei nicht nur Tektonik und Setzung im Spiel sind, sondern daß das Wasser in den einzelnen Teilgebieten der Marsch verschieden hoch auflief. Welche Faktoren dabei mitwirkten, kann nur durch intensive Erforschung begrenzter Siedlungsräume geklärt werden. Die vieljährigen Untersuchungen des Niedersächsischen Landesinstituts für Marschenund Wurtenforschung, z. B. im Lande Wursten und in der Marsch der unteren Ems, lieferten Befunde von vielen Siedlungsplätzen innerhalb kleiner Siedelräume, und durch geologische und paläobotanische Untersuchungen war es möglich, die natürliche Landschaft in der Umgebung der Siedlungen zu rekonstruieren.

Die Flachsiedlungen der älteren vorrömischen Eisenzeit in der Flußmarsch links der unteren Ems liegen alle auf dem aus schluffigen Tonen aufgebauten Uferwall der Ems (Behre 1970; vgl. Brandt 1972: Abb. 1). Die unterschiedliche Höhenlage der Wohnniveaus dürfte nicht auf Setzung oder Tektonik zurückzuführen sein, sondern auf die Lage der Siedlungen innerhalb der natürlichen Landschaft. Die beiden Siedlungen HatzumBurgstätte (3.3) und Oldendorp-Fuchsgatt (4.1) lagen unmittelbar an der Ems, also in dem Bereich stärkster Sedimentation, wo aber bei Überflutungen auch die höchsten Wasserstände auftraten (Louwe-Kooljmans 1976: 126 f.). Die Wohnniveaus dort liegen heute bei $-0,20 /+0,06 \mathrm{~m}$ NN. Tiefere Wohnniveaus fanden sich in der Siedlung Boomborg nahe Hatzum (3.1: -0,90/-0,40 m NN), die ca. $500 \mathrm{~m}$ vom damaligen Emsufer entfernt auf dem Uferwall eines Prieles angelegt worden war, und in der Siedlung Jemgumkloster (1.1: - 0,86/—0,54 m NN), die etwa $8 \mathrm{~km}$ weiter flußaufwärts an der Ems lag. Bei beiden Siedlungsplätzen dürfte sich die Erscheinung ausgewirkt haben, daß in unbedeichter Marsch die Gezeiten zum Landesinneren hin abgeschwächt werden. Diese Interpretation wird gestützt durch die Befunde aus den beiden Siedlungen Jemgum I und Jemgum III $(2.1,2.2)$. Mit Wohnniveaus in der Höhe von $-0,50 /-0,30 \mathrm{~m}$ NN liegen sie zwischen den Werten von Hatzum-Burgstätte und Oldendorp-Fuchsgatt einerseits und Boomborg und Jemgumkloster andererseits. Das entspricht ihrer Lage am Ufer eines sehr 
breiten Prieles, der bei dem heutigen Ort Jemgum (ca. $6 \mathrm{~km}$ flußaufwärts von Hatzum) in die Ems mündete.

Die beiden Phänomene, Stau der Flutwelle nahe der Mündung und Abschwächung der Gezeiten zum Binnenland hin, werden sich auch in anderen Teilen der nordwestdeutschen Marsch in der Weise ausgewirkt haben, daß Siedlungen zur gleichen Zeit in unterschiedlichen Niveaus angelegt wurden.

In zwei frühgeschichtlichen Siedlungen, in Boomborg und Feddersen Wierde, wurden am Ufer eines Prieles Bootslandestege aufgedeckt. Solche Stege waren in etwa auf das Niveau des Mittleren Tidehochwassers eingestellt. Der im Jahre 1969 entdeckte Bootslandesteg in Boomborg (Abb. 2: 3.4), der in die Zeit um Chr. Geb. zu datieren ist, endete am Priel in Höhe von -0,70/-0,55 m NN. Wie hoch die gleichzeitig errichteten Häuser lagen, war infolge jüngerer Störungen nicht genau festzustellen, schätzungsweise bei $+0,30 /+0,40 \mathrm{~m}$ NN (HaArnagel 1969: 92). Der Bootslandesteg der Siedlungsschicht $1 \mathrm{c}$ der Feddersen Wierde (Abb. 2: 19.7), der in der 2. Hälfte des 1. Jh. n. Chr. Geb. gebaut wurde, reichte bis $-0,15 \mathrm{~m}$ NN hinab (HAarnagel 1979: 176). Die Wohnniveaus der zugehörigen Häuser lagen bei $+0,50 /+1,25 \mathrm{~m} \mathrm{NN}$ (Abb. 2: 19.2). Geht man davon aus, $\mathrm{daß}$ die Landestege in Höhe des Mittleren Tidehochwassers und die Wohnniveaus knapp über den Sturmflutpegeln lagen, dann ergibt sich für Boomborg ein Höhenunterschied von $90-100 \mathrm{~cm}$ und für Feddersen Wierde ein Wert von $65-140 \mathrm{~cm}$. Bei der Interpretation dieser Zahlen ist zu berücksichtigen, daß eine in der Flußmarsch gelegene Siedlung wie Boomborg unter extrem hohen Wasserständen länger zu leiden hatte als die unmittelbar an der Meeresküste gelegene Feddersen Wierde, wo das Wasser schneller wieder abfloß als in der Flußmarsch bei Boomborg (vgl. Abb. 2). Aus den Beobachtungen in Boomborg und Feddersen Wierde läßt sich ableiten, daß um Chr. Geb. und im 1. Jh. n. Chr. Geb. die höchsten Fluten dort nicht mehr als etwa $1 \mathrm{~m}$ höher aufliefen als das Mittlere Tidehochwasser.

Abschließend ist festzustellen, daß das Diagramm der ur- und frühgeschichtlichen Wohnniveaus in der Marsch zwischen Ems- und Elbemündung ein sehr differenziertes Bild ergeben hat und neue Fragen aufwirft, die zu beantworten, Aufgabe weiterer Untersuchungen ist.

\section{Verzeichnis der Wohnniveaus in ur- und frühgeschichtlichen Siedlungen in der Marsch zwischen Ems- und Elbemündung}

1.1 Jemgumkloster, Siedlungsschicht 1 (BRANDT 1972)

1.2 Jemgumkloster, Siedlungsschicht 2 (BRANDT 1972)

1.3 Bentumersiel, Siedlungsschicht 1 (BRANDT 1977)

1.4 Jemgumkloster, Siedlungsschicht 3 (BRANDT 1972)

1.5 Jemgumkloster, Siedlungsschicht 4-6 (BRANDT 1972)

1.6 Jemgumkloster, Siedlungsschicht 7 (BRANDT 1972)

2.1 Jemgum I (HAARNAGEL 1957)

2.2 Jemgum III, Siedlungsschicht 1, (Grabungsakten, Nds. Landesinst. f. Marschen- u. Wurtenforschung, Wilhelmshaven)

2.3 Eppingawehr (Schroller 1933; Zylmann 1933: 114 u. 144 f.)

3.1 Boomborg, Siedlungsschicht 1 A (HAARNAGEL 1969)

3.2 Boomborg, Siedlungsschicht 5 (HAARNAGEL 1969)

3.3 Hatzum-Burgstätte (Ältere Eisenzeit) (Grabungsakten, Nds. Landesinst. f. Marschen- u. Wurtenforschung, Wilhelmshaven)

3.4 Boomborg, Siedlungsschicht 6 - Landungssteg (Grabungsakten, Nds. Landesinst. f. Marschen- u. Wurtenforschung, Wilhelmshaven)

3.5 Hatzum-Langwurt (BRANDT 1979)

3.6 Hatzum-Burgstätte (Mittelalter) (BRANDT 1979)

3.7 Alte Boomborg (Grabungsakten, Nds. Landesinst. f. Marschen- u. Wurtenforschung, Wilhelmshaven)

4.1 Oldendorp. Vor dem Fuchsgatt (Brandt \& Behre 1977)

4.2 Klunderborg (Grabungsakten, Nds. Landesinst. f. Marschen- u. Wurtenforschung, Wilhelmshaven) 
5.1 Groothusen, Siedlungsschicht II

5.2 Groothusen, Siedlungsschicht III

5.3 Groothusen, Siedlungsschicht IV

5.4 Groothusen, Siedlungsschicht V

(ReinhaRdt 1965 a: 122-132)

5.5 Groothusen, Siedlungsschicht VI

5.6 Groothusen, Siedlungsschicht VII

6.1 Uttumer Escher, Siedlungsschicht 11

6.2 Uttumer Escher, Siedlungsschicht 2

6.3 Alt-Damhusen, Siedlungsschicht I \}

6.4 Alt-Damhusen, Siedlungsschicht II

(REINHARDT 1965 b: $412 \mathrm{f.)}$

(Reinhardt 1965 a: 132 f.)

7 Visquard (ReinhardT 1965 b: 412 f. u. Grabungsakten, Nds. Landesinst. f. Marschen- u. Wurtenforschung, Wilhelmshaven)

8.1 Middelstewehr, Siedlungsschicht I

8.2 Middelstewehr, Siedlungsschicht II

8.3 Middelstewehr, Siedlungsschicht III

9 Westdorf (Reinhardt 1965 b: 410-412)

10 Nesse (REINHARDT 1965 b: 410-412)

11 Förriesdorf und Zissenhausen (SChrollen 1934; Schütte 1934: 133-136; SChмid 1965: 35)

12 Seeverns (SchüTte 1932; RINK 1932)

13 Langwarden (Grabung 1978, Grabungsakten, Nds. Landesinst. f. Marschen- u. Wurtenforschung, Wilhelmshaven)

14 Niens (Grabung 1979, Grabungsakten, Nds. Landesinst. f. Marschen- u. Wurtenforschung, Wilhelmshaven)

$\left.\begin{array}{l}\text { 15.1 Einswarden, Flachsiedlung } \\ \text { 15.2 Einswarden, 1. Wurtauftragung }\end{array}\right\}$ (SснміD 1957: 50-53)

16 Rodenkirchen, Hahnenknooper Mühle (HAYEN 1972; Grabungsakten, Nds. Landesinst. f. Marschen- u. Wurtenforschung, Wilhelmshaven)

17 Golzwarderwurp (Schütrte \& Rink 1935; SCHмID 1957: 72)

18.1 Barward, Siedlungsschicht 1 (GenRich 1941; Schmid 1957: 53 f.; 1965: 31)

18.2 Fallward, Siedlungsschicht 1 (Stürtz \& Waller 1935; Schmid 1957: 54 f.; 1965: 31)

19.1 Feddersen Wierde, Siedlungshorizont 1 a

19.2 Feddersen Wierde, Siedlungshorizont $1 \mathrm{c}$

19.3 Feddersen Wierde, Siedlungshorizont 2

19.4 Feddersen Wierde, Siedlungshorizont 5

19.5 Feddersen Wierde, Siedlungshorizont 6

19.6 Feddersen Wierde, Siedlungshorizont $7+8$

19.7 Feddersen Wierde, Landungssteg im Siedlungshorizont $1 \mathrm{c}$

20.1 Lüdingworth, Seewurt Osterende (Grabungsakten, Nds. Landesinst. f. Marschen- u. Wurtenforschung, Wilhelmshaven)

20.2 Lüdingworth, Seewurt Wetcke (WaLLER 1934)

21 Ostermoor (BANTELMANN 1960)

22.1 Hodorf, Siedlungsschicht I

22.2 Hodorf, Siedlungsschicht Ia

22.3 Hodorf, Siedlungsschicht IIa/III

23 Ritsch (HaARNaGel 1940: 92-95; SCHMID 1965: $28 \mathrm{f}$.)

24 Barnkrug (HaARnagel 1937 b; 1940: 92-95; Schmid 1965: 29-31)

\section{Schriftenverzeichnis}

Asmus, W.-D. (1949): Neuere Ausgrabungen in der eisenzeitlichen Dorfsiedlung auf der Barward bei Dingen, Kr. Wesermünde. - Hammaburg, 2: 116-130; Hamburg.

Bantelmann, A. (1960): Die kaiserzeitliche Marschensiedlung von Ostermoor bei Brunsbüttelkoog. - Offa, 16, 1957/58: 53-79; Neumünster.

BeHRE, K.-E. (1970): Die Entwicklungsgeschichte der natürlichen Vegetation im Gebiet der unteren Ems und ihre Abhängigkeit von den Bewegungen des Meeresspiegels. - Probleme der Küstenforschung im südlichen Nordseegebiet, 9: 13-47; Hildesheim.

BRANDT, K. (1972) Untersuchungen zur kaiserzeitlichen Besiedlung bei Jemgumkloster und Bentumersiel (Gem. Holtgaste, Kreis Leer) im Jahre 1970. - Neue Ausgrabungen und Forschungen in Niedersachsen, 7: 145-163; Hildesheim.

- (1977): Die Ergebnisse der Grabung in der Marschsiedlung Bentumersiel/Unterems in den Jahren 1971-1973. - Probleme der Küstenforschung im südlichen Nordseegebiet, 12: 1-31; Hildesheim. 
- (1979): Siedlung und Wirtschaft in der Emsmarsch während des frühen und hohen Mittelalters - Historisch-archäologische Untersuchungen. - Probleme der Küstenforschung im südlichen Nordseegebiet, 13: 155-185; Hildesheim.

- \& BenRe, K.-E. (1977): Eine Siedlung der älteren vorrömischen Eisenzeit bei Oldendorf (Unterems) mit Aussagen zu Umwelt, Ackerbau und Sedimentationsgeschehen. - Nachrichten aus Niedersachsens Urgeschichte, 45: 447-458; Hildesheim.

GenRich, A. (1941): Bericht über die Untersuchungen auf der Barward (Gemarkung Imsum, Krs. Wesermünde) - Probleme der Küstenforschung im südlichen Nordseegebiet, 2: 157-170; Hildesheim.

HAarnagel, W. (1937 a): Die frühgeschichtlichen Siedlungen in der schleswig-holsteinischen Elbund Störmarsch, insbesondere die Siedlung Hodorf. - Offa, 2: 31-78, 9 Taf.; Neumünster.

- (1937 b): Bericht von der Grabung in Barnkrug. - Die Kunde, 5, 5: 91-96; Hannover.

- (1940): Die Marschsiedlungen in Schleswig-Holstein und im linkselbischen Küstengebiet. Probleme der Küstenforschung im südlichen Nordseegebiet, 1: 87-98; Hildesheim.

- (1957): Die spätbronze-, früheisenzeitliche Gehöftsiedlung Jemgum b. Leer auf dem linken Ufer der Ems. - Die Kunde, N.F., 8 ,1-2: 1-44; Hannover.

- (1969): Die Ergebnisse der Grabung auf der ältereisenzeitlichen Siedlung Boomborg/Hatzum, Kreis Leer, in den Jahren 1965 bis 1967. - Neue Ausgrabungen und Forschungen in Niedersachsen, 4, 58-97; Hildesheim.

- (1979): Die Grabung Feddersen Wierde. Methode, Hausbau, Siedlungs- und Wirtschaftsformen sowie Sozialstruktur. - Feddersen Wierde II, 2 Bde.; Wiesbaden.

Hayen, H. (1972): Siedlung der späten Bronzezeit und frühen Eisenzeit in der Marsch bei Rodenkirchen, Kr. Wesermarsch. - Nachrichten aus Niedersachsens Urgeschichte, 41: 261 f.; Hildesheim.

Louwe-Kooijmans, L. P. (1976): Prähistorische Besiedlung im Rhein-Maas-Deltagebiet und die Bestimmung ehemaliger Wasserhöhen. - Probleme der Küstenforschung im südlichen Nordseegebiet, 11: 119-143; Hildesheim.

LöBert, H. W.: Die eisenzeitliche Keramik von Hatzum/Boomborg. - Probleme der Küstenforschung im südlichen Nordseegebiet, 14; Hildesheim (im Druck).

Reinhardt, W. (1965 a): Studien zur Entwicklung des ländlichen Siedlungsgebildes in den Seemarschen der ostfriesischen Westküste. - Probleme der Küstenforschung im südlichen Nordseegebiet, 8: 73-148; Hildesheim.

- (1965 b): Wurtengrabungen in Ostfriesland. - Germania, 43: 410-413; Berlin.

RINK, O. (1932): Untersuchungen an der Wurt bei Seeverns in Butjadingen. - Unveröff. Mskr. Nds. Landesinst. f. Marschen- u. Wurtenforschung, Wilhelmshaven.

Schmid, P. (1957): Die vorrömische Eisenzeit im nordwestdeutschen Küstengebiet. - Probleme der Küstenforschung im südlichen Nordseegebiet, 6: 49-120; Hildesheim.

- (1965): Die Keramik des 1. bis 3. Jahrhunderts n. Chr. im Küstengebiet der südlichen Nordsee, 8: 9-46, 25 Taf.; Hildesheim.

Schroller, H. (1933): Eine Siedlungsgrabung bei Eppingawehr, Gemeinde Midlum, Kr. Leer. Die Kunde, Nr. 1, 3/4: 9-10; Hannover.

- (1934): Die Marschenbesiedlung des Jever- und Harlingerlandes. Ein Beitrag zur Küstensenkungsfrage. - Oldenburger Jahrbuch, 37/1933: 160—187; Oldenburg.

SChüтte, H. (1932): Geologische Auswertung der Wurtuntersuchung in Seeverns. - Unveröff. Mskr. Nds. Landesinst. f. Marschen- u. Wurtenforschung, Wilhelmshaven.

- (1934): Der geologische Aufbau des Jever- und Harlingerlandes und die erste Marschbesiedlung. - Oldenburger Jahrbuch, 37/1933: 121-159; Oldenburg.

- \& RiNk, O. (1935): Warften über einer Flachsiedlung in Golzwarderwurp. - Oldenburger Jahrbuch, 38/1934: 141-179; Oldenburg.

Sindowski, K.-H. \& STREIF, H. (1974): Die Geschichte der Nordsee am Ende der letzten Eiszeit und im Holozän. - In: Woldstedt, P./Duphorn, K.: Norddeutschland und angrenzende Gebiete im Eiszeitalter. 3. Aufl., 411-431; Stuttgart.

Streif ,H. (1971): Stratigraphie und Faziesentwicklung im Küstengebiet von Woltzeten in Ostfriesland. - Beihefte zum Geologischen Jahrbuch, 119, 59 S.; Hannover.

Stürtz, E. \& Waller, K. (1935): Die Fallward. Ergebnisse einer Ausgrabung auf einer Wurt im Lande Wursten. - Mannus, 27: 223-238; Leipzig.

WalLeR, K. (1934): Eine Wurtuntersuchung im Lande Hadeln. - Jb. Männer vom Morgenstern, 26: $97-110$; Bremerhaven.

WaterbolK, H. T. \& Boersma, J. W. (1976): Bewoning in voor- en vroeghistorische tijd. - In: Formsma, W. J. et al.: Historie van Groningen. Stad en Land: 11-74; Groningen.

Wildvang, D. (1938): Die Geologie Ostfrieslands. - Abhandlungen des Preußischen Geologischen Landesamtes, N.F., H. 181, 211 S.; Berlin.

Zylmann, P. (1933): Ostfriesische Urgeschichte. - Darstellungen aus Niedersachsens Urgeschichte, 2: 187 S.; Hildesheim/Leipzig. Manuskript eingegangen am 2. 5. 1980. 